Article

\title{
Two-Objective Optimization of a Kaplan Turbine Draft Tube Using a Response Surface Methodology
}

\author{
Riccardo Orso ${ }^{1}$, Ernesto Benini ${ }^{1, *} \mathbb{C}$, Moreno Minozzo ${ }^{2}$, Riccardo Bergamin ${ }^{2} \mathbb{D}$ and \\ Andrea Magrini ${ }^{1}$ (D) \\ 1 Department of Industrial Engineering, University of Padua, Via Venezia 1, 35131 Padua, Italy; \\ riccardo.orso@studenti.unipd.it (R.O.); andrea.magrini@phd.unipd.it (A.M.) \\ 2 ZECO S.r.l., via Astico 52/c, 36030 Fara Vicentino, Italy; moreno.minozzo@zeco.it (M.M.); \\ riccardo.bergamin@zeco.it (R.B.) \\ * Correspondence: ernesto.benini@unipd.it
}

Received: 20 August 2020; Accepted: 16 September 2020; Published: 18 September 2020

\begin{abstract}
The overall cost of a hydropower plant is mainly due to the expenses of civil works, mechanical equipment (turbine and control units) and electrical components. The goal of a new draft tube design is to obtain a geometry that reduces investment costs, especially the excavation ones, but the primary driver is to increase overall machine efficiency, allowing for a reduced payback time. In the present study, an optimization study of the elbow-draft tube assembly of a Kaplan turbine was conducted. First, a CFD model for the complete turbine was developed and validated. Next, an optimization of the draft tube alone was performed using a design of experiments technique. Finally, several optimum solutions for the draft tube were obtained using a response surface technique aiming at maximizing pressure recovery and minimizing flow losses. A selection of optimized geometries was subsequently post-checked using the validated model of the entire turbine, and a detailed flow analysis on the obtained results made it possible to provide insights into the improved designs. It was observed that efficiency could be improved by $1 \%$ (in relative terms), and the mechanical power increased by $1.8 \%$ (in relative terms) with respect to the baseline turbine.
\end{abstract}

Keywords: Kaplan turbine; draft tube optimization; CFD analysis; DOE; response surface

\section{Introduction}

In recent decades, environmental policies have been oriented towards reducing energetic dependence on fossil fuels, leading to renewed and increased investments in the hydroelectric sector; such investments have been primarily directed to optimizing both new designs and existing installations, thus allowing for more efficient plants and reduced payback times. Kaplan hydro turbines have been adopted for a long time to deal with efficient energy production in the range of high specific speeds [1]. The use of movable vaned distributors and runner blades, in fact, allows to limit incidence flow losses and achieve high conversion efficiency over a wide range of flow rates, from $40 \%$ to $120 \%$ of the nominal value. The main drawback of Kaplan turbines is that their size increases as the flow rate gets higher, and the cost attributed to civil works becomes more and more substantial.

In these machines, the draft tube is an essential component as it makes it possible to recover a significant fraction of the kinetic energy leaving the runner by conversion into static pressure. This, in turn, leads to creating an additional suction head downstream of the runner, which allows to increase the effective head that the runner is able to deliver [2]. In addition, the draft tube plays a fundamental role in determining turbine efficiency since the height at which the tube is installed contributes to a large percentage of the total net head that can be recovered [3]. 
Moreover, it is one of the most challenging parts to analyze from a fluid flow perspective due to the interaction of many complex flow features, such as unsteadiness, turbulence, separation, streamline curvature, secondary flow, swirl, and vortex breakdown [4].

Studies involving Kaplan turbine components made use of both steady and unsteady Reynolds-Averaged Navier-Stokes (RANS), the Scale-Adaptive Simulation (SAS) and hybrid RANS-LES (Large Eddy Simulations) models, such as the Zonal Large Eddy Simulation (ZLES) or the Detached Eddy Simulations (DES). Jošt reported an improvement in characteristic parameter predictions for a Kaplan turbine using unsteady models and ZLES [5]. Marjavaara [4] performed steady and unsteady CFD simulations of a draft tube using RANS and DES. The characteristic quantities and the local flow features obtained were highly dependent on the model and the inflow boundary conditions. These models can accurately predict turbulent phenomena in the draft tube but require very large computational resources. As such, their adoption in optimization studies seems, today, still infeasible, while validated RANS approaches offer a very good compromise between accuracy and computational effort [3,5]. Li [6] used steady RANS to study the hydraulic performance of a Kaplan turbine's components. Brijkishore [7] evaluated the influence of runner solidity using the k- $\Omega$ SST turbulence model in steady computations. Liu [8] carried out transient analyses on the runner lifting-up during load rejection using the same RANS model.

Past studies on draft tube optimizations were limited in terms of representativeness. In fact, while the draft tube can be acceptably optimized as an isolated component around its design point, therefore neglecting the interference effects with the turbine runner, the performances of optimal configurations have not been post-checked using the entire turbine model for a posteriori validation of the complete turbine installation. Lyutov [9] described a coupled optimization of runner and draft tubes, where their geometries were varied simultaneously, allowing to achieve a $0.3 \%$ higher efficiency gain compared to single-runner optimization. Ciocan [10] presented a draft tube optimization from part to full load, using a parameterized velocity field at the tube entry based on a swirl-free velocity profile for the runner. Eisinger [11] reported an automatic draft tube optimization, but no coupling of the inflow conditions with the attached runner was present. Puente [12] employed RANS simulations with the $k-\varepsilon$ turbulence model for the same purpose, using normalized velocity profiles at the duct inlet, derived from previous turbine simulations. Sosa [13] analyzed the influence of draft tube modification using a steady computational model with a single-channel guide vane and runner connected to the tube via a mixing plane. The geometric modifications based on a two-parameter description were meant to represent erosion or material addition in the wall. Schiffer [14] performed steady-state simulations of a complete model, including distributors, runners, and a draft tube, at several operating points. The tube was then manually adapted to optimize the turbine's efficiency while keeping construction costs low.

In the present paper, a fully 3D Kaplan turbine RANS CFD model was first built and validated against available experimental data in terms of net head, flow rate, efficiency, and mechanical power.

Following this, the pressure and velocity distribution at the draft tube inlet were extracted from previous computations. Finally, a CFD model that contained only the draft tube and its outlet extension was created and used in the optimization process. Unlike previous studies, the optimization was carried out based on two-objectives which are of prominent interest to draft tube designers, i.e., its pressure recovery and its total pressure loss coefficient. Also, a noteworthy improvement with respect to past studies relies on the verification of the obtained results: in fact, once the optimal draft tube geometries had been found, they were tested back using the full machine CFD model to find out their impact on the turbine's efficiency and to analyze the sources of draft tube losses in detail. 


\section{Materials and Methods}

\subsection{Draft Tube Performance}

The datum, or baseline, draft tube was connected to a Kaplan turbine runner installed in a $576 \mathrm{~kW}$ hydraulic power plant located in the Adda river (Italy), as depicted in Figure 1a. The original geometry adopted for the elbow draft tube is schematically represented in Figure $1 \mathrm{~b}$.

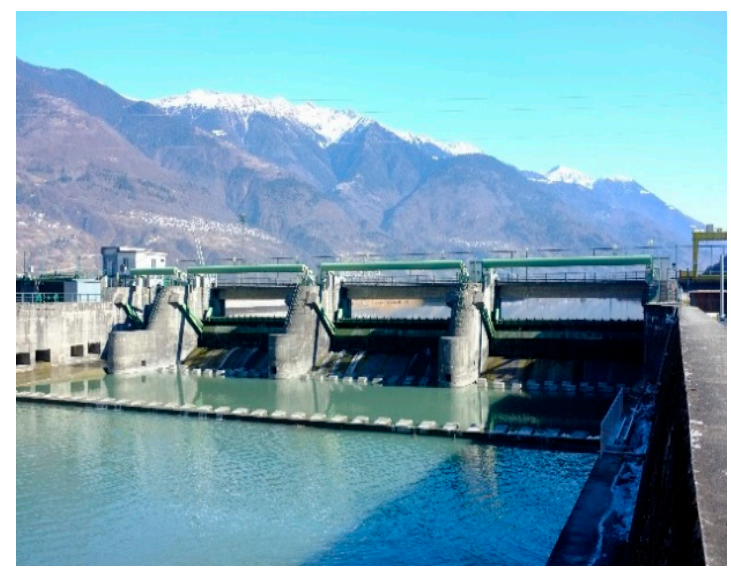

(a)
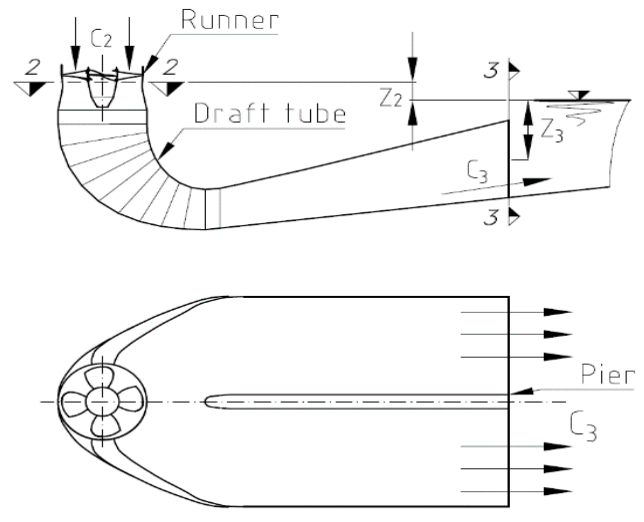

(b)

Figure 1. Original power plant: (a) overall external view; (b) baseline elbow-draft tube geometry.

As mentioned above, the draft tube behaves as a diffuser acting to recover part of the kinetic energy from Section 2 to 3, according to its prescribed area distribution along the equivalent centerline.

Two coefficients are used here to quantify the performance of a draft tube, namely a pressure recovery factor $(C p)$ and a drag coefficient $(C d)$, respectively defined as:

$$
C_{p}=\frac{p_{3}-p_{2}}{\frac{1}{2} \rho c_{2}^{2}} ; \quad C_{d}=\frac{p_{t o t-2}-p_{t o t-3}}{\frac{1}{2} \rho c_{2}^{2}}
$$

where $p$ is the static pressure and $p_{t o t}$ the total pressure; subscripts 2 and 3 refer to the station downstream of the runner and on the draft tube outlet, respectively; $\rho$ is the water density; $c$ is the absolute velocity.

The values of these coefficients are strictly related to the area distribution and are notoriously conflicting in such a way that the more intense the diffusion is (high $C p$ ), the bigger the head loss is (high $C d$ ) that might occur as a result of flow separation, secondary losses, etc. Therefore, a good draft tube features the highest possible $C p$ along with the minimum achievable $C d$. These two coefficients were used as objective functions in the multi-objective optimization study described later.

\subsection{CFD Model Setup and Validation}

The steady-state RANS CFD model of the complete turbine adopted included complete spiral casing with stay vanes, guide vanes, and runner and draft tubes (Figure 2). The final grids were chosen based on a mesh sensitivity analysis and their details and statistics are provided in Table 1 . The influence of mesh size on the draft tube performance parameters is shown in Figure 3, where a limited sensitivity to the grid size can be observed, starting from the third level, having $1.8 \mathrm{M}$ cells. As for boundary conditions, a total pressure was defined at the inlet of the spiral casing and a static pressure equal to $0 \mathrm{~Pa}$ (relative to the atmospheric pressure value of $1 \mathrm{~atm}$ ) was set at the outlet of the draft tube.

The total pressure at the inlet boundary corresponded to the total energy that the plant could process and was calculated from the net head and the flow rate that the turbine was subjected to. The flow solver was Ansys CFX 19.2. Since the runner was not modelled as a rotating mesh, 
a multiple-reference-frame approach was chosen, and a mixing-plane condition was set both at the distributor-runner and runner-draft tube interfaces. Among all the turbulence models (all the variants of both $k-\varepsilon$ and k- $w$ available in Ansys CFX 19.2 were actually tested), it was observed that the k- $w$ SST [15] provided the most accurate predictions, but it was also the one that required the highest computational effort; for this reason, the k- $w$ SST was used in off-loop analyses on the complete machine model, while the standard $\mathrm{k}-\varepsilon$ was used in the optimization due to its cheaper requirement. A physical timescale corresponding to $1^{\circ}$ of runner rotation and a high-resolution scheme were set.

In order to assure improved numerical stability, 50 iterations were run at first using first-order schemes. These results were adopted as initial values for the high-resolution scheme.

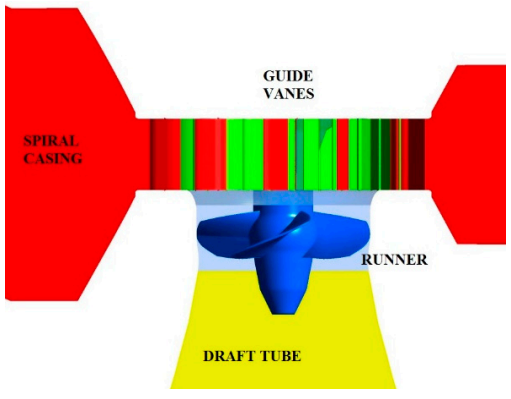

(a)

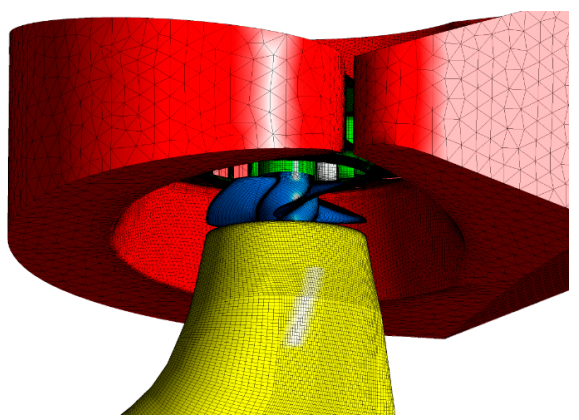

(c)

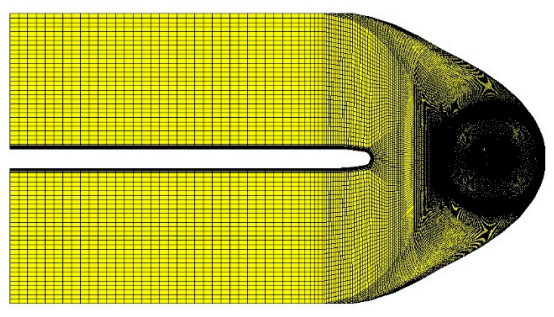

(e)

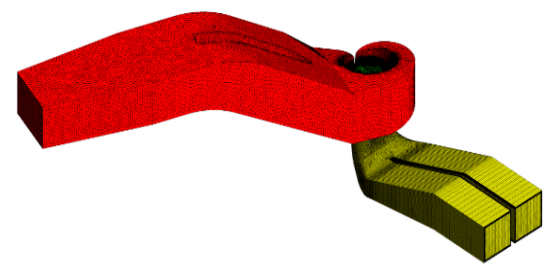

(b)

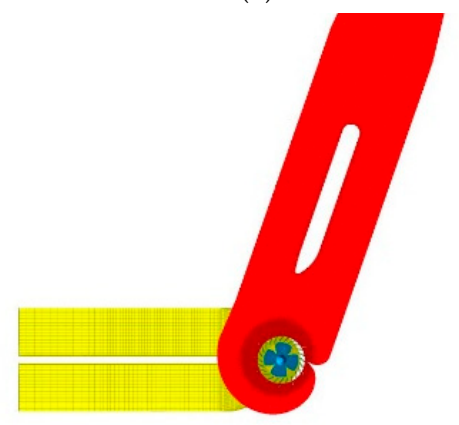

(d)

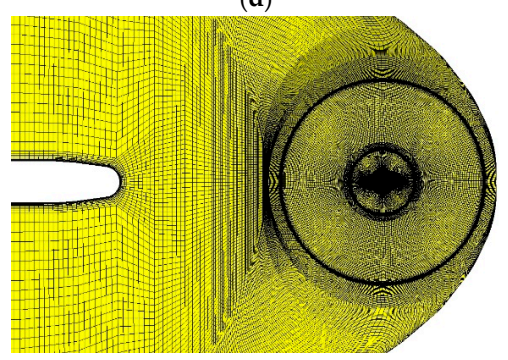

(f)

Figure 2. Turbine computational domains: (a) close up of the runner domain in meridional view; (b) 3D view of the overall domain; (c) close up of the computational mesh in the region close to the runner/draft tube interface; (d) top view of the overall domain; $(\mathbf{e}, \mathbf{f})$ details of the draft tube mesh.

Table 1. Details of the grids used in the subdomains.

\begin{tabular}{cccccccc}
\hline Subdomain & $\begin{array}{c}\text { Meshing } \\
\text { Tool }\end{array}$ & $\begin{array}{c}\text { Mesh } \\
\text { Type }\end{array}$ & $\begin{array}{c}\text { Number } \\
\text { of Nodes }\end{array}$ & $\begin{array}{c}\text { Number of } \\
\text { Elements }\end{array}$ & $\begin{array}{c}\text { Average } \\
\text { Y+ }\end{array}$ & $\begin{array}{c}\text { Number of Boundary } \\
\text { Layer Prism }\end{array}$ & $\begin{array}{c}\text { Boundary Layer } \\
\text { Growth Ratio }\end{array}$ \\
\hline Spiral casing with stay vanes & ICEM-CFD & Tetra & $2,580,807$ & $7,685,535$ & 62 & 6 & 1.2 \\
\hline Guide vanes & Turbogrid & Hexa & $1,939,968$ & $1,781,112$ & 95 & 40 & 1.1 \\
\hline Distributor ring & ICEM-CFD & Hexa & 478,720 & 462,672 & 28 & 12 & 1.5 \\
\hline Runner & Turbogrid & Hexa & $1,023,320$ & 964,180 & 110 & 50 & 1.05 \\
\hline Draft tube with extension & ICEM-CFD & Hexa & $1,882,944$ & $1,842,398$ & 65 & 25 & 1.2 \\
\hline
\end{tabular}




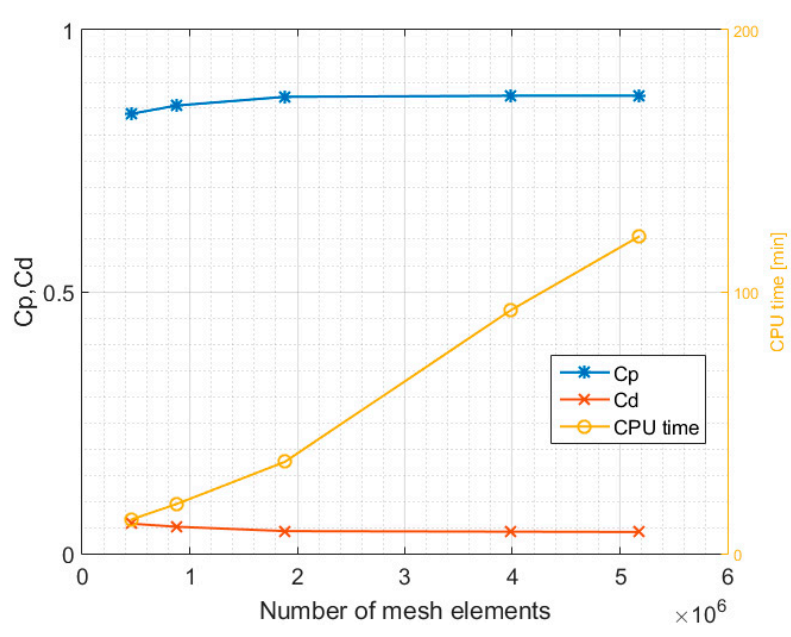

Figure 3. Mesh independency analysis for the draft tube.

During the convergence runs, several variables of interest were monitored, such as flow rate, efficiency, static pressure at draft tube inlet, and mechanical power output. Final results were available after 3000 iterations on average and almost $72 \mathrm{~h}$ of CPU calculations. All calculations were performed on a server equipped with Intel ${ }^{\circledR}$ Xeon ${ }^{\circledR}$ CPU x5650 processors using parallel solutions on 10 multiple cores. Convergence was assessed when all variables of interest showed a variation lower than $0.08 \%$.

Results from both experiments and simulations are presented in Figure 4. All the experimental data (net head, flow rate, efficiency, and mechanical power) were taken in compliance with the European IEC EN 60041:1991 standard [16] and included the appropriate uncertainty bandwidth. Power and efficiency data from CFD were calculated as follows:

$$
P=M \cdot \omega ; \eta=\frac{P}{\rho Q g H}
$$

where $M$ is the total torque on the runner blades, $\omega$ is the rotational speed, $Q$ is the flow rate, and $H$ is the net head. As can be observed in Figure 4, the power and efficiency data were within the experimental measurement uncertainty range. The steady RANS CFD model set up appeared, therefore, to be able to reproduce the machine performance with sufficient accuracy and acceptable computational time. From this validated model, the runner-draft tube interface conditions, that is, the distributions of the $u$, $v, w$ (respectively tangential, absolute, and relative velocity components), were extracted to be used as inlet boundary conditions during the optimization process.

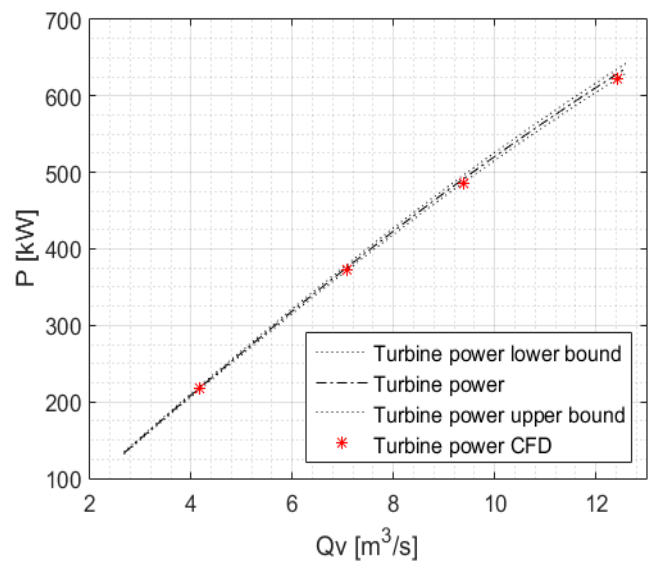

(a)

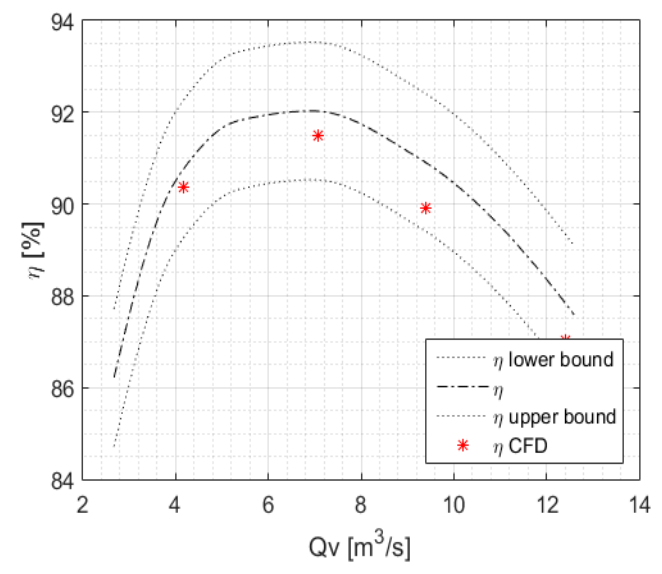

(b)

Figure 4. Validation results: (a) power data; (b) efficiency. 


\subsection{Draft Tube Geometry and Parameterization}

The draft tube geometry of Kapan turbines is complicated by the different shapes of its inlet and outlet sections. The first is circular due to the interface with the runner, while the second is often rectangular. Moreover, the draft tube features a $90^{\circ}$ elbow to minimize excavation costs and to improve powerhouse compactness. As a result, a large number of design parameters is necessary to provide successful geometry parameterization. In the present paper, the draft tube was parameterized using a mean line and a number of cross-sections stacked along it.

The mean line was designed to be composed by a first straight section, made up of two segments, one related to the divergent cone, and one to the cylindrical section; a second curvilinear line was related to the elbow shape and controlled by a three-point Bézier polygon; finally, a segment was connected to the last section of the draft tube.

The first straight part of the mean line was described by two parameters-the two segments' lengths. For the Bézier polygon, four additional variables were introduced-the coordinates of the polygon-points (the coordinates of the first Bézier polygon point were derived from the first straight part). The last parameter was the length of the last straight segment. In total, 7 variables were involved in the parameterization of the mean line. The area distribution was managed using a 4 th-degree polynomial as a function of the mean line coordinate $x$ :

$$
A(x)=P 1 \times\left(P 2 \times a x^{4}+b x^{3}+c x^{2}+d x\right)+e
$$

In Figure 5, the area distribution of the original geometry is represented using dots along with the polynomial interpolation. From the original geometry, it was possible to obtain the polynomial coefficients $(a, b, c, d, e)$ and use $\mathrm{P} 1$ and $\mathrm{P} 2$ as decision variables.

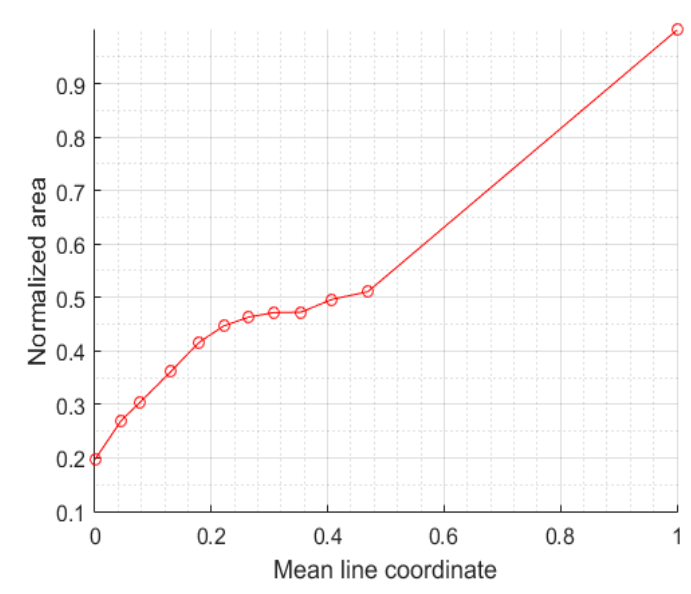

(a)

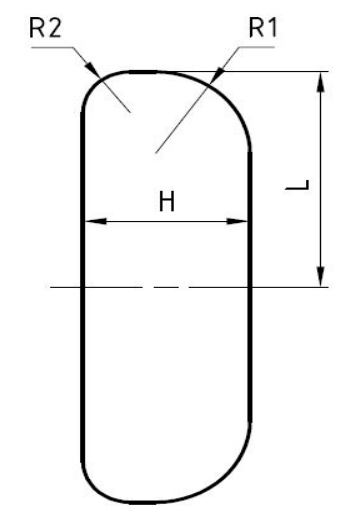

(b)

Figure 5. Draft tube geometry definition: (a) area distribution; (b) shape of a generic cross section.

Overall, the draft tube was parameterized using 9 decision variables. In order to avoid the need for further parameters to account for the shape change from inlet to exit sections, the geometric similarity with the baseline tube was preserved, keeping the geometric ratios $\mathrm{R} 1 / \mathrm{L}, \mathrm{R} 2 / \mathrm{L}$, and $\mathrm{H} / \mathrm{L}$ for each section along the mean line equal to those of the datum.

\subsection{Optimization: Problem Formulation and Tools}

As previously mentioned, the optimization study was conducted on the draft tube alone by taking the inlet boundary conditions from the full machine model at the runner-draft tube interface. As a result, the flow domain included the runner discharge cone along with the runner tube interface 
(which form the inlet boundary), the first divergent part, the elbow, the last divergent part, and the draft tube prolongation leading to the outlet section.

The optimization was multi-objective, with the purpose of maximizing $C p$ and minimizing $C d$ at the same time using a Pareto approach. As for the constraints, the optimization had to lead to solutions that were interchangeable with the baseline case currently installed without any modifications regarding the runner. As a result, during the entire procedure, the baseline inlet and runner cone (both belonging to the runner) remained fixed. A further constraint was related to the global draft tube depth which affects the cost of civil works. The outlet depth could vary in all directions but with the limit of always remaining with a certain margin under the free water surface of the tailrace.

A response surface methodology for the optimization tool was used [17]. The first step was a design space exploration using design of experiments (DOE) in order to understand how the design parameters were related to each other and which were the most significant ones. A "central composite design" (CCD) DOE technique was adopted, which provided a screening set of 150 samples to determine the overall trends of the metamodel. The latter was built using the genetic aggregation method [18] and the Pareto optimal solutions were finally obtained (Figure 6a). A check-in-the-loop of optimal solutions was necessary to evaluate the response surface goodness using CFD (Figure 6b); the best-so-far optimum individuals from the response surface were taken and verified using a dedicated CFD simulation, then inserted back in the DOE, which, in turn, updated the response surface from which a new Pareto front was obtained. The loop was kept running until a stable and invariant Pareto optimal set was determined.

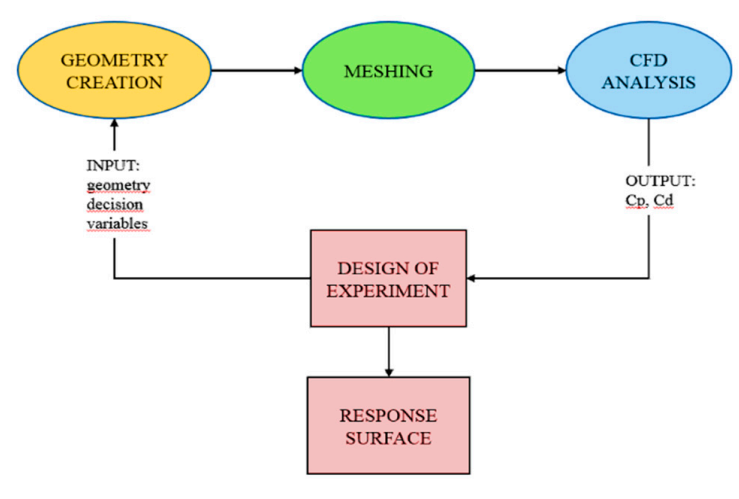

(a)

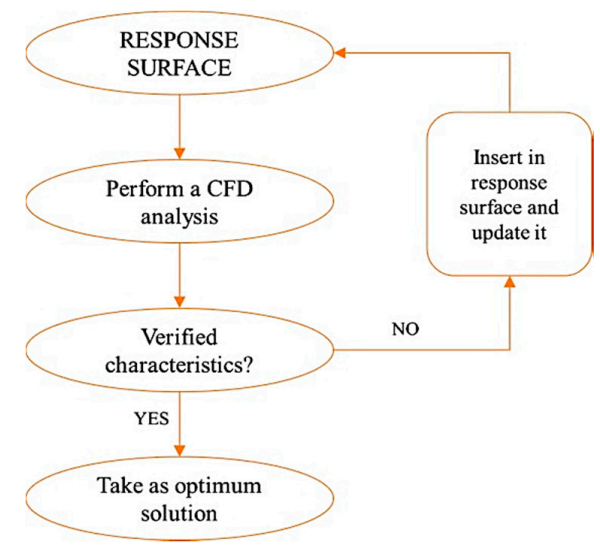

(b)

Figure 6. Optimization procedure: (a) general loop; (b) response surface update workflow.

It is worth mentioning that during the loop iterations, the response surface method allowed for an adaptive refinement in the search regions around maximum $C_{P}$ and minimum $C_{D}$ so as to make it possible to have a wider and more uniform Pareto front.

\section{Results and Discussion}

The final Pareto front is depicted in Figure 7, from which a subset of relevant configurations could be extracted: the baseline solution is marked with a black diamond, while the optimum $C_{D}$, the optimum $C_{P}$, and a good compromise between the $C_{D}$ and $C_{P}$ are marked using a red triangle, a yellow circle, and a green square, respectively. Dominated individuals are indicated as other points in the figure. The performance data of optimized individuals are given in Table 2. The solution corresponding to the minimum $C_{D}$ (Candidate 1 ) featured a reduction count $\Delta C_{D}=-0.0254$ (i.e., -2.54 percentage point reduction), while the one corresponding to the maximum $C_{P}$ (Candidate 3) showed $\Delta C_{P}=+0.0574$ (i.e., +5.7 percentage point improvement). 


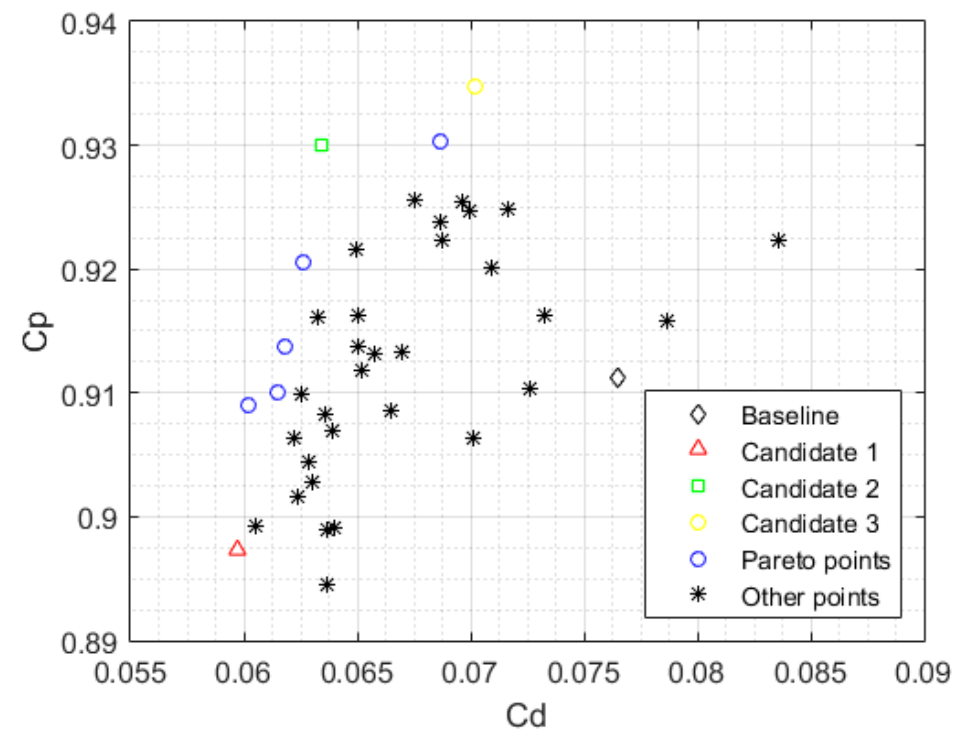

Figure 7. Optimization results: Pareto solutions.

Table 2. Optimization results: Pareto solutions.

\begin{tabular}{ccc}
\hline Performance Data & $\boldsymbol{C}_{\boldsymbol{d}}$ & $\boldsymbol{C}_{\boldsymbol{p}}$ \\
\hline Baseline & 0.0851 & 0.9186 \\
\hline Candidate 1 & 0.0597 & 0.8974 \\
\hline Candidate 2 & 0.0634 & 0.9300 \\
\hline Candidate 3 & 0.0702 & 0.9347 \\
\hline
\end{tabular}

\subsection{Optimal Draft Tube Configurations}

In Figure 8, the area distribution along the mean line coordinate for the Pareto optimal subset of solutions compared to the baseline one is shown. It can be seen that optimal solutions exhibited a much lower increase in area distribution from the beginning of the draft the tube (mean line coordinate $>0.05$, see Figure $8 b$ ); this is beneficial to limit adverse phenomena during flow diffusion in this zone as confirmed, also, by the distribution of $\mathrm{Cd}$ versus the mean line coordinate (Figure 10a), particularly visible in the case of Candidate 1.

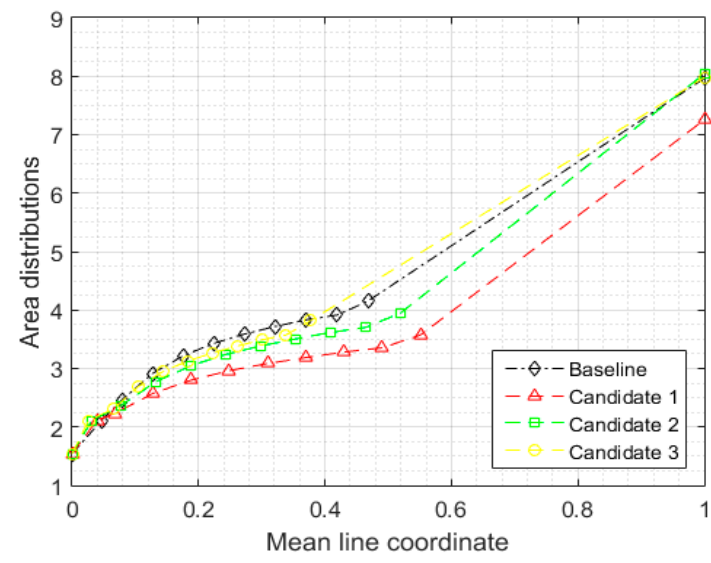

(a)

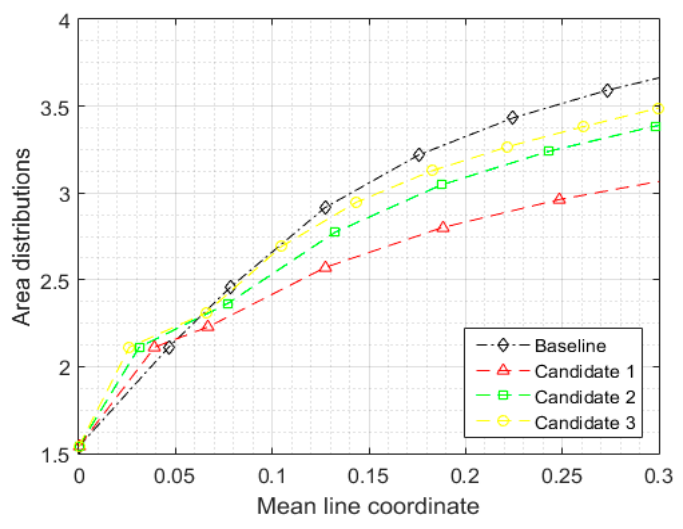

(b)

Figure 8. Optimization results: (a) area distribution (b) close up in the range $x \in[0-0.3]$. 
In Figure 9, a detailed comparison between the optimal solutions and the baseline is presented. For the first draft tube part (until the $20 \%$ of the mean line), very similar characteristics can be observed, while the most remarkable differences are visible in the elbow and in the last part.

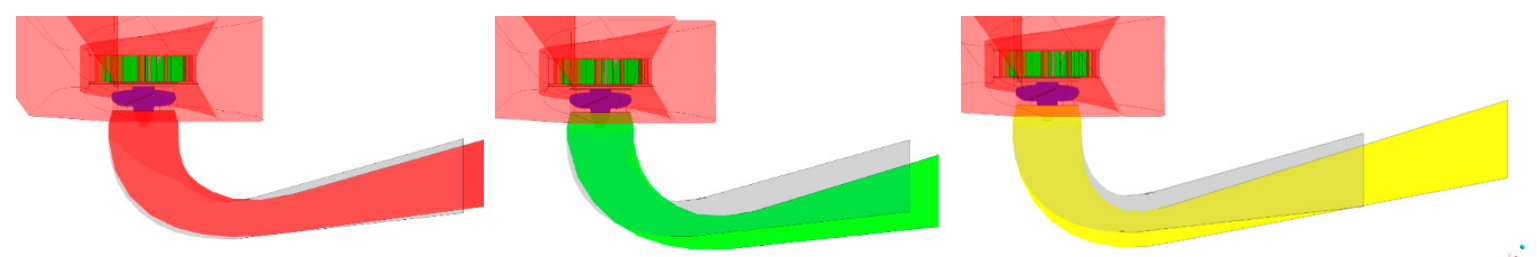

Figure 9. Comparison among geometries in meridional view: Baseline (grey); Candidate 1 (red); Candidate 2 (green); Candidate 3 (yellow).

Candidate 1 had a very similar geometry compared to the baseline, while Candidate 2 featured an elbow with a much higher radius of curvature. Candidate 3 , on the other hand, had much larger dimensions than the baseline and this has a major impact on the excavation costs. As it will be seen later, Candidate 2 was the best in performance and this is due to the shape of the elbow, a fact that emphasizes the importance of producing a good design in the region where a big part of the diffusion is realized by the draft tube.

\subsection{Post-Check Validation on Full Machine and Result Transposition}

A number of Pareto optimal draft tube geometries were obtained from the optimization and were subsequently analyzed, a posteriori, by connecting them into the CFD model of the entire machine in order to assess their influence on critical plant characteristics, such as mechanical power and efficiency. In Figure 10 , both the $C_{d}$ and $C_{p}$ of the optimal draft tube geometries computed using the full CFD model of the turbine are plotted as functions of the mean line coordinate.

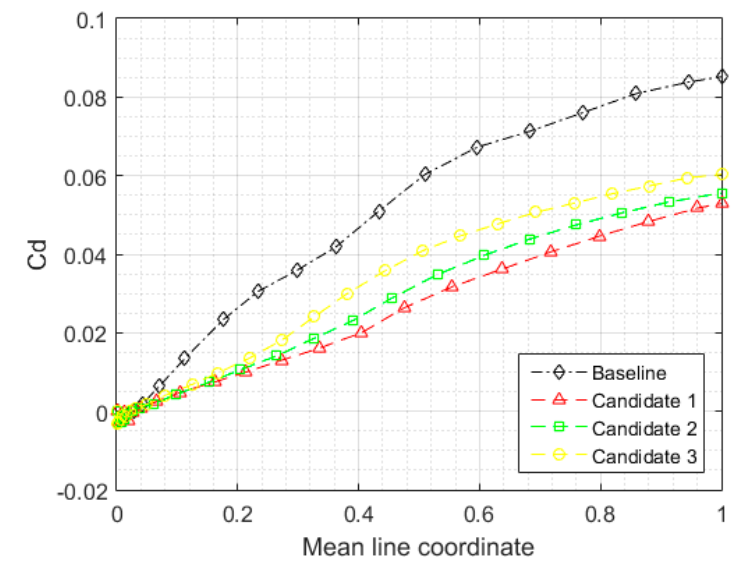

(a)

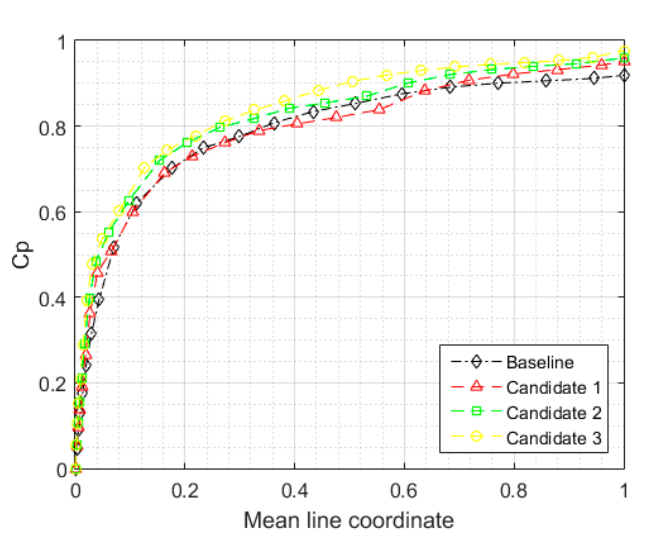

(b)

Figure 10. Post-check results on draft tube performance using full machine model: (a) $C_{d}$ distribution; (b) $C_{p}$ distribution.

The CFD analyses performed on the full machine returned different values for the turbine performance figures, especially for the net head and mass flow rate (Table 3). For a better comparison, a result transposition was carried out according to the European IEC EN 60041:1991 standard [16]. In particular, the mass flow rate and the power output data were transposed using the baseline net head. This was acceptable since the condition $0.99<\sqrt{ } \mathrm{H} / \sqrt{ } \mathrm{H}^{\prime}<1.01$ on head $\mathrm{H}^{\prime}$ compared to baseline $\mathrm{H}$ was met for each candidate. Optimal candidate solutions led to greater power and efficiency values compared to the baseline. In particular, Candidate 2 featured an increment of $1.8 \%$ on the produced power and $1 \%$ in efficiency ratio. 
Table 3. Full machine CFD results (transposed based on baseline CFD results).

\begin{tabular}{ccccccc}
\hline CFD & $\mathbf{Q}^{\prime} \mathbf{v}\left[\mathbf{m}^{3} / \mathbf{s}\right]$ & $\mathbf{H}[\mathbf{m}]$ & $\boldsymbol{\eta} / \eta_{\text {baseline }}$ & $\eta$ Variation $[\%]$ & $\mathbf{P}_{\mathbf{P}}$ baseline & P Variation [\%] \\
\hline Baseline & 9.251 & 5.88 & 100 & - & 100 & - \\
\hline Candidate 1 & 9.286 & 5.88 & 100.57 & +0.57 & 100.957 & +0.96 \\
\hline Candidate 2 & 9.323 & 5.88 & 101.00 & +1.00 & 101.790 & +1.79 \\
\hline Candidate 3 & 9.281 & 5.88 & 100.53 & +0.53 & 100.856 & +0.86 \\
\hline
\end{tabular}

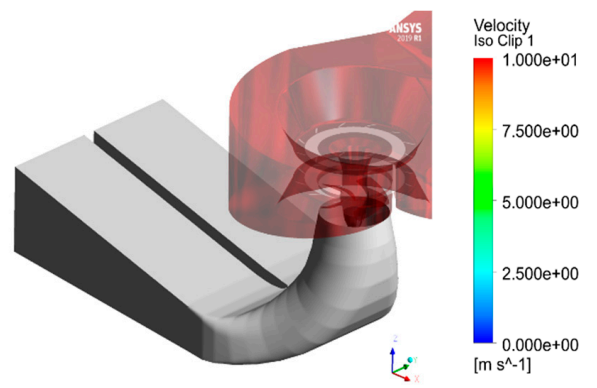

(a)

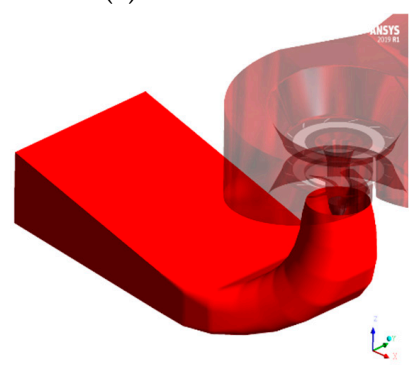

(b)

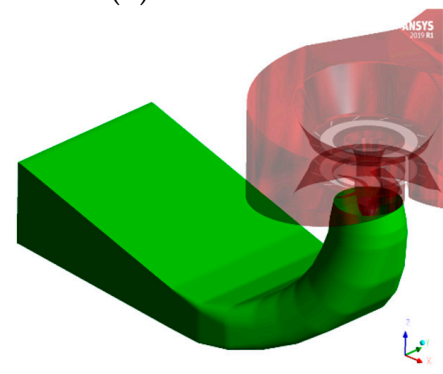

(c)

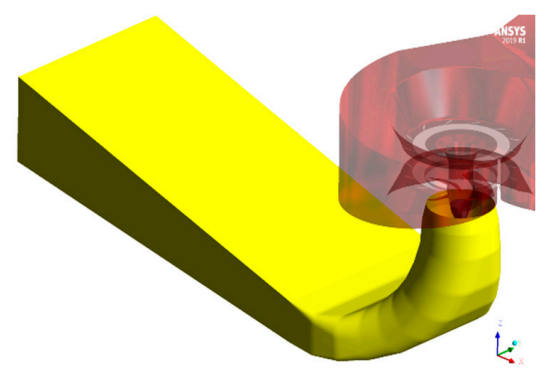

(d)

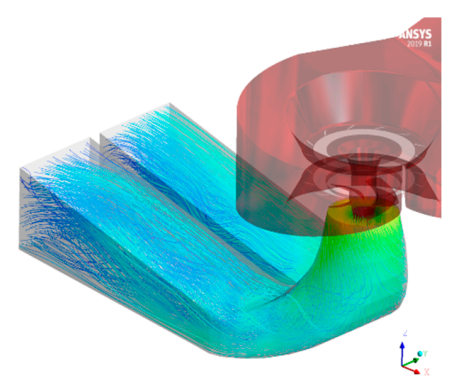

$\left(\mathbf{a}^{\prime}\right)$
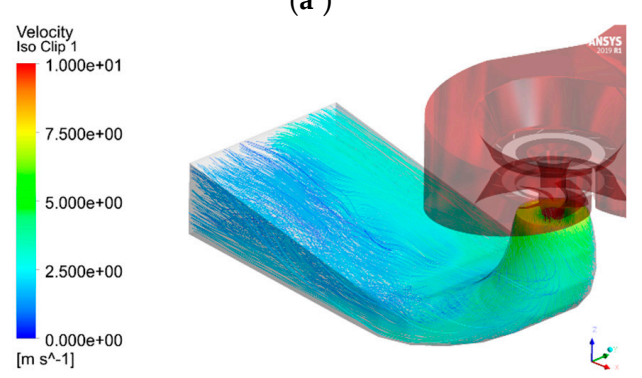

$\left(\mathbf{b}^{\prime}\right)$
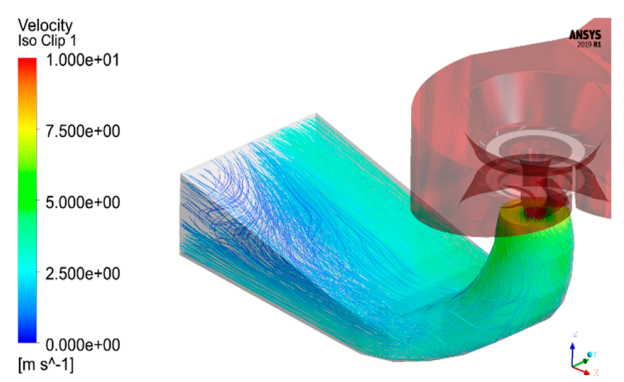

$\left(c^{\prime}\right)$

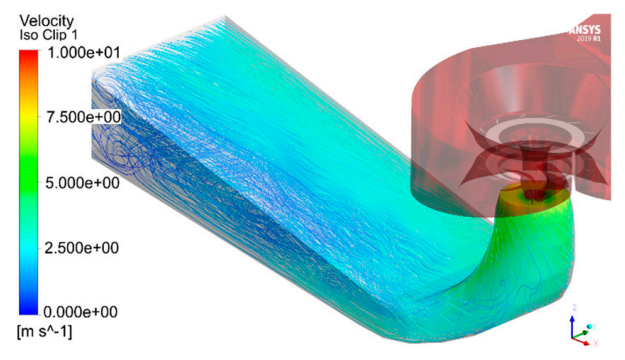

$\left(d^{\prime}\right)$

Figure 11. Comparison of geometries (left column), and streamlines colored by velocity (right column): $\left(\mathbf{a}, \mathbf{a}^{\prime}\right)$ Baseline; $\left(\mathbf{b}, \mathbf{b}^{\prime}\right)$ Candidate $1 ;\left(\mathbf{c}, \mathbf{c}^{\prime}\right)$ Candidate $2 ;\left(\mathbf{d}, \mathbf{d}^{\prime}\right)$ Candidate 3. 
From a geometrical point of view (Figure 9), Candidates 1 and 2 were not very different from the baseline, although they clearly exhibited an elbow shape having a larger radius of curvature, which eventually caused the exit stations of both the elbows and the draft tubes to be placed further downstream with respect to the baseline. Additionally, Candidate 3 featured the same global tendencies but with a much longer draft tube, which, eventually, would lead to higher installation costs. In Figure 11 a $3 \mathrm{D}$ view of the several solutions is presented, with a comparison of the several streamlines. It can be seen that candidate 1 has the most uniform flow, a fact confirmed by his $C_{d}$ value (Figure 9).

From a fluid dynamic standpoint, it is apparent that the largest part of the pressure recovery took place in the first part of the diffuser (Figure 10b), which confirms the importance of having a very accurate design of the region upstream of the elbow.

As a matter of fact, approximately $70 \%$ of the pressure recovery is realized in the first $20 \%$ length of the diffuser. This also held true for the total pressure losses which were primarily generated in this region (see $C_{d}$ behavior in Figure 10, where the largest slope is evidenced in the $C_{d}$ distribution in the first $20 \%$ length of the baseline geometry); localized losses accumulated all along the draft tube mean line, finally leading to an overall large $C_{d}$ value in the baseline. Such an observation confirms what was previously pointed out in [5] and [13]. Furthermore, by looking at the total pressure distribution on consecutive station cuts along the draft tube (Figure 12, right column), it is evident that the rotational region at the lowest total pressure in the core of the discharge flow was of a much lower intensity in the optimized solutions compared to the baseline, despite the latter having a separation wall in the middle of the diffuser. In fact, the analyses carried out showed that the vortex rope greatly influences the draft tube's performance, creating instabilities and turbulent zones in the flow field.

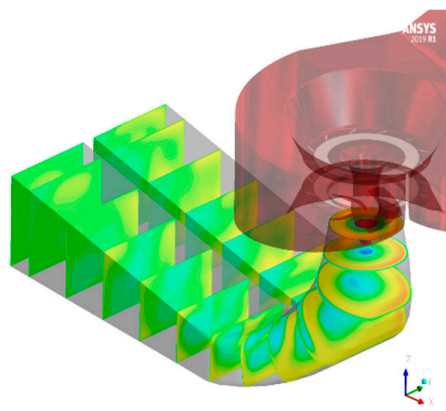

(a)

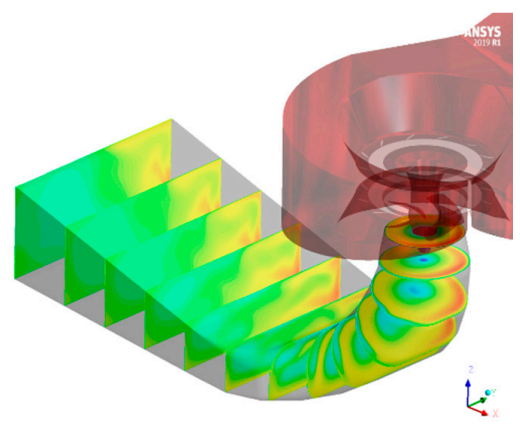

(c)
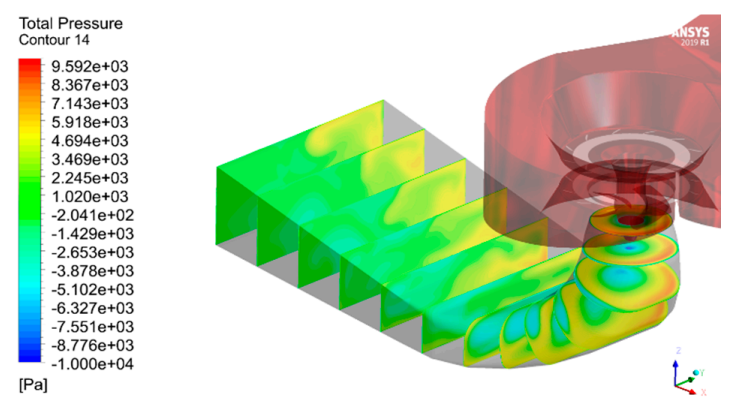

(b)

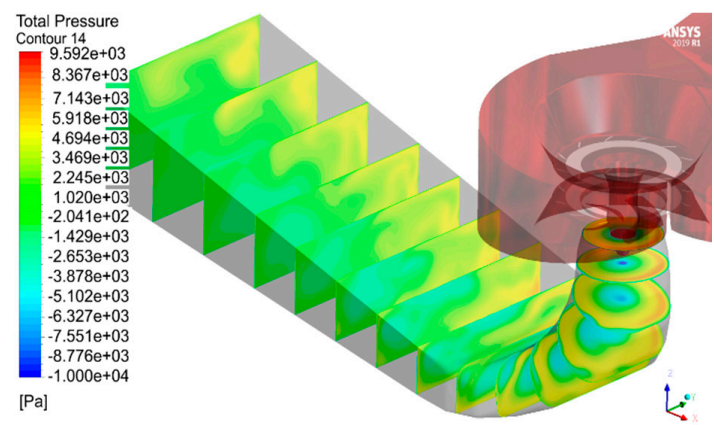

(d)

Figure 12. Comparison of relative total pressure distributions: (a) Baseline; (b) Candidate 1; (c) Candidate 2; (d) Candidate 3 .

\section{Conclusions}

A validated CFD model of a draft tube of a Kaplan turbine was successfully implemented and used for a multi-objective optimization based on the construction of a response surface. The shape 
optimization involved only the draft tube domain, where a one-way coupling with the runner was included in the form of a prescribed velocity distribution extracted from the full-component baseline model.

Two Pareto optimal designs have been extracted from the final set which outperformed the baseline. Regarding the performance of the draft tube alone, the optimum $C_{D}$ featured a reduction count of -0.0254 ( -2.54 percentage point reduction), while the optimum $C_{P}$ showed an incremental count of +0.0574 (i.e., +5.7 percentage point improvement) with respect to the baseline geometry.

A post-check carried out including the optimized draft tubes in the overall turbine confirmed the optimization trends, although the best improvements in terms of delivered hydraulic power-in the case where the overall turbine was considered-have been registered, including a third type of diffuser which exhibited an increment of $1.8 \%$ in the power produced.

The three best candidates overall were slightly deeper than the baseline in terms of excavation dimensions, so a cost/benefit assessment will be needed to determine which is the most appropriate draft tube for a given plant. In addition, future work will be related to extending the investigation to more operating points by performing a multi-objective multi-point optimization, where the Pareto solutions will account for the different conditions at the tube inflow along the turbine operating line.

Author Contributions: Conceptualization, E.B. and R.O.; methodology, R.O. and E.B.; software, R.O.; validation, R.O., M.M. and R.B.; formal analysis and writing-original draft preparation, R.O.; writing—review and editing, E.B.; supervision, E.B.; review and editing, A.M. All authors have read and agreed to the published version of the manuscript.

Funding: This research received no external funding.

Conflicts of Interest: The authors declare no conflict of interest.

\section{References}

1. Kovalev, N.N. Hydroturbines Design and Construction; Israel Program for Scientific Translation: Jerusalem, Israel, 1965.

2. Krivchenko, G.I. Hydraulic Machines Turbines and Pumps, 2nd ed.; CRC-Press: Boca Raton, FL, USA, 1994.

3. McNabb, J.; Devals, C.; Kyriacou, S.A.; Murry, N.; Mullins, B.F. CFD based draft tube hydraulic design optimization. In Proceedings of the 27th IAHR Symposium on Hydraulic Machinery and Systems, Montreal, QC, Canada, 22-26 September 2014; Désy, N., Ed.; IOP Publishing: Bristol, UK, 2014.

4. Marjavaara, B.D. CFD Driven Optimization of Hydraulic Turbine Draft Tubes using Surrogate Models. Ph.D. Thesis, Lulea University of Technology, Lulea, Sweden, 2006.

5. Jošt, D.; Škerlavaj, A.; Lipej, A. Improvement of Efficiency prediction for a Kaplan Turbine with Advanced Turbulence Models. Stroj. Vestn. J. Mech. Eng. 2014, 60, 124-134. [CrossRef]

6. Li, Y.; Liu, Q. Analysis of hydraulic performance for Kaplan turbine components based on CFD simulation. IOP Conf. Ser. Earth Environ. Sci. 2020, 510, 022038. [CrossRef]

7. Brijkishore; Khare, R.; Prasad, V. Performance Evaluation of Kaplan Turbine with Different Runner Solidity Using CFD. In Advanced Engineering Optimization through Intelligent Techniques; Advances in Intelligent Systems and Computing; Springer: Singapore, 2020; Volume 949, pp. 757-767.

8. Liu, K.; Yang, F.; Yang, Z.; Zhu, Y.; Cheng, Y. Runner lifting-up during load rejection transients of a Kaplan turbine: Flow mechanism and solution. Energies 2019, 12, 4781. [CrossRef]

9. Lyutov, A.E.; Chirkov, D.V.; Skorospelov, V.A.; Turuk, P.A.; Cherny, S.G. Coupled Multipoint Shape Optimization of Runner and Draft Tube of Hydraulic Turbines. J. Fluids Eng. 2015, 137, 111302. [CrossRef]

10. Ciocan, T.; Susan-Resiga, R.; Muntean, S. Improving draft tube hydrodynamics over a wide operating range. J. Hydraul. Res. 2016, 54, 74-89. [CrossRef]

11. Eisinger, R.; Ruprecht, A. Automatic Shape Optimization of Hydro Turbine Components Based on CFD. 2002. Available online: http://kwk.ihs.uni-stuttgart.de/fileadmin/IHS-Startseite/veroeffentlichungen/v2001_05.pdf (accessed on 10 June 2020).

12. Puente, L.R.; Reggio, M.; Guibault, F. Automatic Shape Optimization of a Hydraulic Turbine Draft Tube. IOP Conf. Ser. Earth Environ. Sci. 2018, 136, 012019.

13. Sosa, J.B.; Urquiza, G.; Garcìa, J.C.; Castro, L.L. Computational fluid dynamics simulation and geometric design of hydraulic turbine draft tube. Adv. Mech. Eng. 2015, 7, 1-11. [CrossRef] 
14. Schiffer, J.; Benigni, H.; Jaberg, H. An analysis of the impact of draft tube modifications on the performance of a Kaplan turbine by means of computational fluid dynamics. Proc. Inst. Mech. Eng. Part C J. Mech. Eng. Sci. 2017, 232, 1937-1952. [CrossRef]

15. ANSYS CFX. Theory Guidev 19.3. Available online: https://ansyshelp.ansys.com/account/secured?returnurl= /Views/Secured/corp/v193/cfx_thry/cfx_thry.html (accessed on 15 July 2020).

16. IEC 60041:1991. In Field Acceptance Tests to Determine the Hydraulic Performance of Hydraulic Turbines, Storage Pumps and Pump-Turbines, 3rd ed.; International Standard 1991-11; International Electrotechnical Commission: Geneva, Switzerland, 1991.

17. Kim, K.-Y.; Samad, A.; Benini, E. Design Optimization of Fluid Machinery; Wiley: Hoboken, NJ, USA, 2019.

18. Salem, M.B.; Roustant, O.; Gamboa, F.; Tomaso, L. Universal Prediction Distribution for Surrogate Models. 2015. Available online: https://arxiv.org/pdf/1512.07560.pdf (accessed on 10 June 2020).

C 2020 by the authors. Licensee MDPI, Basel, Switzerland. This article is an open access article distributed under the terms and conditions of the Creative Commons Attribution (CC BY) license (http://creativecommons.org/licenses/by/4.0/). 